\title{
A SHORT PROOF AND GENERALIZATION OF A MEASURE THEORETIC DISJOINTIZATION LEMMA
}

\author{
JOSEPH KUPKA
}

\begin{abstract}
This paper presents general conditions under which a subfamily may be selected from an infinite family of nonnegative, finitely additive measures such that this subfamily has the same cardinality as the original family, and such that the members of this subfamily are, in a certain sense, dis jointly supported. The generalized continuum hypothe$s$ is is required for the general result, but not for a special case of this result which had previously been obtained by Rosenthal, and for which the present techniques yield a much shorter proof.
\end{abstract}

This paper contains a short proof of a lemma about finitely additive measures which was first obtained by Rosenthal [2, Lemma 1.1, p. 16] in connection with a number of results on Banach spaces. Some illustration is then provided of the type of generalization which may be obtained with the present techniques.

We shall understand an ordinal number to be a set and not an order type (see $[1, \S 4.3$, p. 19]). As we shall rely heavily upon the axiom of choice, it will be sufficient for our purposes to define a cardinal number to be an initial ordinal number, i.e. an ordinal number which cannot be put into a oneto-one correspondence with any of its members. If $\Gamma$ is any set, let $|\Gamma|$ denote the cardinality of $\Gamma$, by which we mean the unique cardinal number which can be put into a one-to-one correspondence with $\Gamma$; let $c f(\Gamma)$ denote the cofinality of $|\Gamma|$, by which we mean the smallest cardinal number $\kappa$ such that $|\Gamma|$ contains a cofinal subset of cardinality $\kappa$; and let $P(\Gamma)$ denote the power set of $\Gamma$, by which we mean the set of all subsets of $\Gamma$.

To simplify notation we have trivially reworded Rosenthal's lemma, which now follows.

1. Lemma. Let $\Gamma$ be an infinite set, let $\left\{\mu_{x}: x \in \Gamma\right\}$ be a family of nonnegative finitely additive measures defined on all subsets of $\Gamma$, and assume

Received by the editors June 25, 1973.

AMS (MOS) subject classifications (1970). Primary 28A10, 28-01, 04A20, 04-01; Secondary 46B99.

Key words and phrases. Finitely additive measures, cardinality. 
that $\sup \left\{\mu_{x}(\Gamma): x \in \Gamma\right\}$ is finite. Then, for all $\epsilon>0$, there exists a set $X \subset \Gamma$ such that $|X|=|\Gamma|$, and such that $\mu_{x}(X \sim\{x\})<\epsilon$ for all $x \in X$.

Proof. Assume that, for some $\epsilon>0$, no such set $X$ exists. Since $\Gamma$ is infinite, it is a well-known consequence of the axiom of choice that $|\Gamma|=$ $|\Gamma \times \Gamma|\left[3,(2.2)\right.$, p. 417], and hence that we have $\Gamma=\bigcup\left\{x_{\gamma}: \gamma \in \Gamma\right\}$, where the $X_{\gamma}$ are pairwise disjoint, and where $\left|X_{\gamma}\right|=|\Gamma|$ for all $\gamma \in \Gamma$. We may now select an index $\gamma_{0} \in \Gamma$ such that $\mu_{x}\left(\Gamma \sim X_{\gamma_{0}}\right) \geq \epsilon$ for all $x \in X_{\gamma_{0}}$. (For otherwise we could select, for all $\gamma \in \Gamma$, an $x_{\gamma} \in X_{\gamma}$ such that $\mu_{x y}\left(\Gamma \sim X_{y}\right)<\epsilon$. The set $X=\left\{x_{y}: \gamma \in \Gamma\right\}$ would then satisfy the conclusions of the Lemma, contrary to assumption.)

If this procedure is repeated with $X_{\gamma_{0}}$ in place of $\Gamma$, and if it is iterated for at most finitely many steps, then the uniform boundedness of the $\mu_{x}$ is seen to be violated. $\square$

Our generalization of this lemma is based upon a transfinite iteration of the procedure of the first paragraph of the above proof. In place of $\mu_{x}$ we shall consider $N_{x}$, the collection of sets of $\mu_{x}$-measure zero, and in fact it will suffice to assume only that $N_{x}$ is hereditary, i.e. that every subset of a member of $N_{x}$ is also a member of $N_{x}$. Define $\delta(x)$ to be the smallest cardinal number $\delta$ such that every pairwise disjoint family of " $x$-nonnull" sets (i.e. sets which are $\notin N_{x}$ ) has cardinality $\leq \delta$. (Thus, in Lemma 1 , we have $\delta(x) \leq \boldsymbol{N}_{0}$ for all $x \in \Gamma$.)

2. Theorem. Assume the generalized continuum hypothesis. Let $\Gamma$ be an infinite set, let $\left\{N_{x}: x \in \Gamma\right\}$ be a family of hereditary subsets of $P(\Gamma)$, and assume that there exists a cardinal number $\kappa<c f(\Gamma)$ such that $\delta(x)<\kappa$ for all $x \in \Gamma$. Then there exists a set $X \subset \Gamma$ such that $|X|=|\Gamma|$, and such that $X \sim\{x\} \in N_{x}$ for all $x \in X$.

Remark. In particular, whenever $c f(\Gamma) \geq \boldsymbol{N}_{2}$, it is consistent with the axioms of set theory to replace "< $\epsilon$ " by " $=0$ " in Lemma 1 .

Proof. Assume that no such set $X$ exists. Since $\Gamma$ is infinite, it is a well-known consequence of the generalized continuum hypothesis that $|\Gamma|=$ $|G|$, where $G$ denotes the set of functions with domain $\kappa$ and codomain $\Gamma$ $\left[1, \$ 36.1\right.$, p. 162]. To simplify notation we shall treat the $N_{x}$ as subsets of $P(G)$.

We now define a function $x^{*} \in G$ such that $\delta\left(x^{*}\right) \geq \kappa$, contrary to hypothesis. Let $\alpha \in \kappa$ be fixed, and assume by transfinite induction that $x^{*}(\beta)$ has been defined for all $\beta<\alpha$. Let $G_{a}=\left\{x \in G: x(\beta)=x^{*}(\beta)\right.$ for all $\beta<\alpha\}$, and observe that $G_{\alpha}=\bigcup\left\{X_{\gamma}: \gamma \in \Gamma\right\}$, where $X_{\gamma}=\left\{x \in G_{a}: x(\alpha)=\gamma\right\}$ 
for all $\gamma \in \Gamma$. The argument of Lemma 1 is applicable, and we define $x^{*}(\alpha)$ $=\gamma_{0}$, where $\gamma_{0}$ is any member of $\Gamma$ such that $G_{a} \sim X_{\gamma_{0}} \notin N_{x}$ for all $x \in$ $X_{\gamma_{0}}$. Clearly, then, we have $\delta\left(x^{*}\right) \geq \kappa$, as desired.

3. Example. Assume that $\Gamma=c f(\Gamma)$, and, for all $x \in \mathrm{I}^{*}$, let $N_{x}$ be the collection of those subsets of $\Gamma$ which are disjoint from the predecessors of $x$. Then we have $\delta(x)=|x|<|\Gamma|=c f(\Gamma)$ for all $x \in \Gamma$, but it is easily shown that any set $X \subset \Gamma$ which satisfies the conclusions of Theorem 2 can contain at most a single point.

In particular, if $\Gamma=\kappa_{0}$ or $\kappa_{1}$, a trivial elaboration of this example will illustrate that, in general, the " $\epsilon$ " of Lemma 1 cannot be changed to " $=0$ " when $c f(\Gamma)=\boldsymbol{N}_{0}$ or $\boldsymbol{\kappa}_{1}$.

To round out the picture we present a sample set of circumstances under which the hypotheses of Theorem 2 may be (slightly) relaxed.

4. Proposition. Assume that each of the $N_{x}$ of Theorem 2 is closed under the formation of finite unions, and that it contains every subset of $\Gamma$ which has cardinality $<|\Gamma|$. Then, provided that $\delta(x)<c f(\Gamma)$ for all $x \in \Gamma$, there exists a set $X \subset \Gamma$ such that $|X|=|\Gamma|$, and such that $X \in N_{x}$ for all $x \in \Gamma$.

Proof. By [3, Theorem 1, p. 451] (cf. [2, Proposition, p. 23]), there exists a set $S \subset P(\Gamma)$ such that $|S|>|\Gamma|$, such that $|E|=|\Gamma|$ for all $E \in S$, and such that $|E \cap F|<|\Gamma|$ whenever $E$ and $F$ are distinct members of $S$. It is easily established that, for all $x \in \Gamma$, there must be (strictly) fewer than $c f(\Gamma)(\leq|\Gamma|)$ many sets $E \in S$ such that $E \notin N_{x}$. It follows that there must exist at least one (and in fact $>|\Gamma|$ many) $X \in S$ such that $X \in N_{x}$ for all $x \in \Gamma$.

5. Example. Assume that $|\Gamma|=c f(\Gamma)$, and, for all $x \in \Gamma$, let $N_{x}$ be the collection of those subsets of $\Gamma$ which have cardinality $<|\Gamma|$. Then we have $\delta(x)=|\Gamma|=c f(\Gamma)$ for all $x \in \Gamma$, whereas no set $X \in N_{x}$ has $|X|=|\Gamma|$.

\section{REFERENCES}

1. H. Bachmann, Transfinite Zahlen, Ergebnisse der Math. und ihrer Grenzgebiete, Heft 1, Springer-Verlag, Berlin, 1955. MR 17,134。

2. H. P. Rosenthal, On relatively disjoint families of measures, with some applications to Banach space theory, Studia Math. 37 (1970), 13-36. MR 42 \#5015.

3. W. Sierpiński, Cardinal and ordinal numbers, 2nd rev. ed., Monografie Mat., vol. 34, PWN, Warsaw, 1965. MR 33 \#2549. 